\title{
Comment on Wang et al.: One-stage posterior focus debridement, interbody graft using titanium mesh cages, posterior instrumentation and fusion in the surgical treatment of lumbo-sacral spinal tuberculosis in the aged
}

\author{
Haibo $\mathrm{Li}^{1,2}$ • Qingyi Dong ${ }^{3}$
}

Received: 4 April 2016 /Accepted: 11 April 2016/Published online: 25 April 2016

(C) SICOT aisbl 2016

Dear Editor,

It was with great interest that I read the study entitled "Onestage posterior focus debridement, interbody graft using titanium mesh cages, posterior instrumentation and fusion in the surgical treatment of lumbo-sacral spinal tuberculosis in the aged" by Wang and colleagues published online in March 2016 in International Orthopaedics [1]. In this paper, the authors report that one-stage posterior surgery was an effective treatment for aged patients with lumbo-sacral spinal tuberculosis. It is an interesting study. Nevertheless, we have several queries which we would like to communicate with the authors.

In general, anti-tuberculosis chemotherapy is the mainstay in the management of spinal tuberculosis. However, major adverse reactions to anti-tuberculosis drugs can cause significant morbidity, and compromise treatment regimens for tuberculosis. Adverse effects of chemotherapy of tuberculosis contained hepatitis, abdominal discomfort, hyperuricemia, peripheral neuritis, retrobulbar neuritis and so on. In addition, there is a higher incidence of adverse anti-tuberculosis drug reactions in elderly TB patients [2]. The predisposing factors for drug toxicity include genetic causes, advanced age, extent of the disease, female gender, nutritional status and excessive doses of the drugs. In this series, the majority of patients ( $70.6 \%$ ) were with comorbidities such as diabetes which could cause diabetic nephropathy,

Qingyi Dong

dongqy030911@163.com

1 The State Key Laboratory of Medical Genetics, School of Life Sciences, Central South University, Changsha, Hunan 410078, China

2 Department of Ophthalmology, Xiangya Hospital, Central South University, Changsha, Hunan 410008, China

3 Institute of Pediatrics, The Second Xiangya Hospital, Central South University, Changsha, Hunan 410011, China diabetic neuropathy, diabetic retinopathy and other severe associated complications. The authors only monitored the blood test and hepatic function of the patients at the follow up. It may lead to ignore other side-effects of anti-tuberculosis drugs like hyperuricemia, and/or aggravate the diabetes associated complications. Is it necessary to use HRZE for about 18 months for treatment of spinal tuberculosis in the aged? To be mentioned, in the authors' previous study about the surgical efficacy of elderly patients with lumbo-sacral tuberculosis, they recommended combination of isoniazid. rifampin and pyrazinamide for more than 18 months to treat spinal tuberculosis after surgery [3].

Additionally, there was a slip of the pen in the result line 3 . "The surgical incisions of both groups were healed without chronic infection, fistula formation, and recurrence." This series didn't divide into two group in the study. There was a citing mistake at patient data line 16. The Fig. 1 a-d did not contains hematologic and pathological examinations.

\section{Compliance with ethical standards}

Conflict of interest The authors declare that they have no conflict of interest.

\section{References}

1. Wang Y-X, Zhang H-Q, Liao W, Tang M-X, Guo C-F, Deng A, Wu J-H, Liu J-Y (2016) One-stage posterior focus debridement, interbody graft using titanium mesh cages, posterior instrumentation and fusion in the surgical treatment of lumbo-sacral spinal tuberculosis in the aged. Int Orthop 1-8

2. Arbex MA, Varella MDCL, Siqueira HRD, Mello FAFD (2010) Antituberculosis drugs: drug interactions, adverse effects, and use in special situations-part 1: first-line drugs. J Bras Pneumol 36:626-640

3. Zhang H-Q, Lin M-Z, Ge L, Li J-S, Wu J-H, Liu J-Y (2012) Surgical management by one-stage posterior transforaminal lumbar debridement, interbody fusion, and posterior instrumentation for lumbo-sacral tuberculosis in the aged. Arch Orthop Trauma Surg 132:1677-1683 